\title{
THE IMPACT OF DISTRACTION ON AN INTERSECTION CROSSING ASSIST SYSTEM
}

\author{
Ensar Becic, Christopher Drucker, Michael Manser, \& Max Donath \\ University of Minnesota \\ Minneapolis, Minnesota, USA \\ Email: ebecic@gmail.com
}

\begin{abstract}
Summary: The current study examines the impact of drivers' use of an in-vehicle intersection crossing assist system under demanding cognitive load conditions. The use and adherence to the assist system is examined through intersection crossing driving performance measures. Furthermore, the impact of distraction is examined for younger and older drivers. The results suggest a more conservative approach to the crossing of rural intersections when using the assist system, a finding which was not altered by cognitive load.
\end{abstract}

\section{INTRODUCTION}

In-vehicle systems designed to assist a driver while navigating the ever increasing complexity of our environment are becoming commonplace. A variety of early warning systems alert a driver to a potential collision (Kramer, Cassavaugh, Horrey, Becic \& Mayhugh, 2007) and lane departure warnings aid drivers in lane keeping (Blaschke, Breyer, Färber, Freyer \& Limbacher, 2009). The technological advancements that led to improvements in transportation safety and comfort, as an unintended consequence also increased the potential for an in-vehicle distraction (e.g., cell-phones, navigation). Substantial research has shown a negative impact of these secondary activities on driving performance (Strayer, Drews \& Johnston, 2003, Strayer, Drews \& Crouch, 2006). Since distracted drivers are slower to respond to sudden events, the primary goal of a driver assist/warning system (e.g., side object detection) becomes capturing drivers' attention, thus enhancing theirs perceptual abilities and ameliorating the cost due to distraction (see Kramer et al.).

While a warning system may increase the likelihood of detection of sudden events (e.g., pedestrian encroaching onto the street) in situations when driver is engaged in a secondary task, what kind of impact does a secondary task have on driver assist systems which do not warn a driver about an immediate threat, but rather present traffic information to the driver? An example of one such system is a Cooperative Intersection Collision Avoidance System-Stop Sign Assist (CICAS-SSA), proposed by Preston, Storm, Donath and Shankwitz (2004). The primary function of this assist system is to help drivers determine an appropriate crossing gap at rural intersections, more specifically, stop-sign controlled intersections with a median. A system such as this is an information display system, rather than a warning system and the impact of a secondary task on drivers' use and adherence to such system has not be adequately explored. While the CICAS-SSA was originally created as a roadside based system, the current study uses an in-vehicle based version of that same system. If the in-vehicle intersection crossing assist system is shown to be beneficial, what impact might an additional cognitive load have on drivers' use of and adherence to that system? If the presence of the assist system results in drivers adopting a more defensive/conservative driving behaviour (e.g., waiting longer to cross, rejecting non-critical gaps), a concurrent secondary task may limit or perhaps further emphasize 
defensive driving. If the benefits of the in-vehicle intersection crossing assist system more directly impact the measures relating to risky crossing (e.g., accepting smaller gaps when crossing, reduced likelihood to stop at a median), performing a concurrent secondary task may reduce those benefits or perhaps completely eliminate them. The current study examines the impact of a secondary task on driver's use of an in-vehicle intersection crossing assist system and the subsequent driving performance.

\section{METHODS}

\section{Participants}

Forty-eight adults, dichotomized into two age groups, participated in this study. The younger age group consisted of participants between the ages of 19 and $28(\mathrm{~N}=24 ; 11$ men; mean=22.1; $\mathrm{sd}=2.52$ years) and the older age group consisted of participants between the ages of 60 and 69 $(\mathrm{N}=24 ; 11$ men; mean=62.2; sd=2.83 years). All participants had a current valid driver's license, normal or corrected-to-normal vision and no history of motion sickness.

\section{Apparatus and Materials}

The present study was conducted in a partial motion-base driving simulator manufactured by Oktal. The simulator consisted of a full vehicle cabin 2002 Saturn SC2 featuring realistic control operations and instrumentations including force feedback steering and braking systems. The visual scene was projected to a high-resolution (2.5 arc-minutes per pixel) five-channel, 210degree forward field of view with rear and side mirror views provided by a rear screen and vehicle-mounted LCD panels. The driving simulator system software replicated the US 52 and CSAW 9 intersection, near Cannon Falls, Minnesota. The major road had four lanes of traffic, two in each direction that were separated by a median. Earlier observations of traffic at that intersection revealed that a gap that drivers rejected $80 \%$ of time was 6.5 seconds, considered to be a critical gap in the current study. The traffic flow in the current study included a large number of gaps for which appropriate crossing is more difficult to determine (4-7 seconds).

The intersection crossing assist system used in the current study was based on the CICAS-SSA sign (Rakauskas, Creaser, Manser, Graving \& Donath, 2009; Creaser, Manser \& Rakauskas, 2008) and represented a simplified version of that sign. More significantly, the CICAS-SSA was moved from its previous location on a roadside, to inside the vehicle. The current sign was divided into two parts. The near lanes representing traffic traveling to the right were overlaid onto the left side mirror (Figure 1a). The far lanes representing traffic traveling to the left were overlaid onto the right side mirror (Figure 1b). When they turned to the left/right to examine the traffic coming from that direction, in the same glance drivers were able to see the information presented by the in-vehicle CICAS-SSA in the left/right side mirror. 
(a)

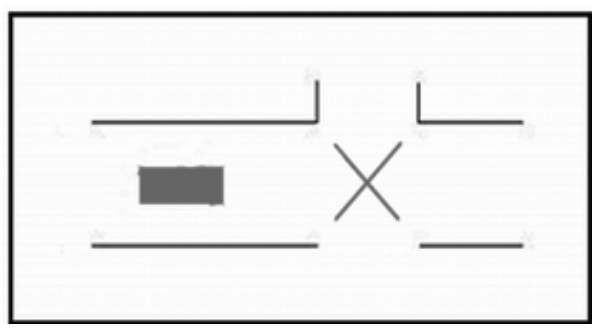

(b)

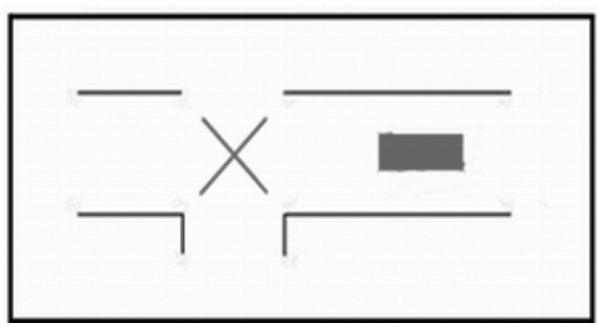

Figure 1. Representation of the in-vehicle CICAS-SSA sign showing an unsafe crossing gap as depicted in the (a) left and (b) right side mirrors

The in-vehicle CICAS-SSA used icons of different colour to indicate the presence of vehicles on the major road. The yellow icon signified a presence of a vehicle, requiring a driver to exercise caution when making a decision to cross (i.e., gap is between 7.5 and 11 seconds). The red icon signified that a vehicle on the major road was too close to the intersection to cross (i.e., gap is less than 6.5 seconds). The blinking yellow icon indicated that the icon was about to turn red.

\section{Procedure}

Driving performance was examined through a trial-based driving task in which participants were asked to approach the intersection, stop at the stop sign, and then cross the intersection in a safe and timely manner. Each trial ended after the participant crossed the intersection. Participants completed a total of 16 trials, half with the CICAS-SSA turned on and the rest with the system turned off. In half of the trials, the participants completed an additional in-vehicle secondary task while driving. The Counting 1-Back task was used to load participants' cognitive resources. In this task, participants heard two, two-digit numbers, presented to them through headphones. They were instructed to provide two answers for each sequence of digits. First, the participants were required to add the last digits from the two numbers they heard. For example, if the participants heard "62, 31", they were required to say “ 3 ” $(2+1=3)$ to answer correctly. Second, the participants needed to determine if their current response was greater or lesser than their previous answer. They were instructed to say their answers out loud which were recorded for later transcription. The participants were instructed to use the in-vehicle CICAS-SSA or not, according to their preference.

\section{RESULTS}

Driving performance measures were examined separately for the crossing of southbound (i.e., stop sign as the starting position) and northbound (i.e., the median as the starting position) lanes. Driving performance was assessed through four measures, some of which more directly examined the probability of inappropriate crossing, and other which are indicators of conservative driving. Adjusted Time-to-contact (measured in seconds) is the modified measure of time-to-contact (ttc) between the nearest cross traffic vehicle and the participant's vehicle when entering the intersection. In this modified ttc measure, the upper value is limited at 6.5 seconds. Likelihood of stopping is the proportion of trials in which the participant made a complete stop. A single-stage maneuver in which drivers do not make a stop at the median is highly correlated with the instances of crashes at this specific intersection. Wait Time is the time between a complete stop at the stop sign or median and the start of the intersection crossing. Rejected noncritical gap is the proportion of times that a driver failed to cross the intersection when the gap 
was greater than the critical gap of 6.5 seconds. Each measure was submitted to a 3-way mixed mode ANOVA with Age (older, younger) as a between-subject factor and Cognitive Load (absent, present) and Sign Presence (Sign on, Sign off) as within-subject factors. From here on, dual-task condition refers to driving while completing the concurrent secondary task, while the single-task condition refers to only performing the driving task.

\section{Wait Time}

Southbound. The wait time measure submitted to a 3-way ANOVA revealed a significant main effect of Sign Presence $(F(1,44)=10.31, p=.002)$. Drivers waited longer to cross the southbound lanes when the sign was turned on (10.3 seconds) compared to when the sign was turned off (7.3 seconds). This analysis also showed a significant interaction between Cognitive Load and Sign Presence $(\mathrm{F}(1,44)=17.51, \mathrm{p}<.001)$. When completing the single-task, drivers waited longer to cross the intersection when the CICAS-SSA sign was turned on compared to the control, Sign off condition $(F(1,44)=25.66, p<.001 ; M=5.6$ and 11.8 seconds for Sign off and Sign on conditions, respectively). However, under dual-task conditions, the wait time duration did not depend on the state of the CICAS-SSA sign $(p>.8)$. This interaction can be viewed from a different point of interest. As illustrated in Figure 2, when the in-vehicle assist system was activated, drivers waited longer to cross in single task condition (i.e., driving only) compared to dual-task $(\mathrm{F}(1,46)=4.81, \mathrm{p}=.033)$. An opposite pattern was found when the assist system was turned off; drivers waited longer to cross when completing a concurrent secondary task $(F(1,44)$ $=7.31, \mathrm{p}=.01)$.

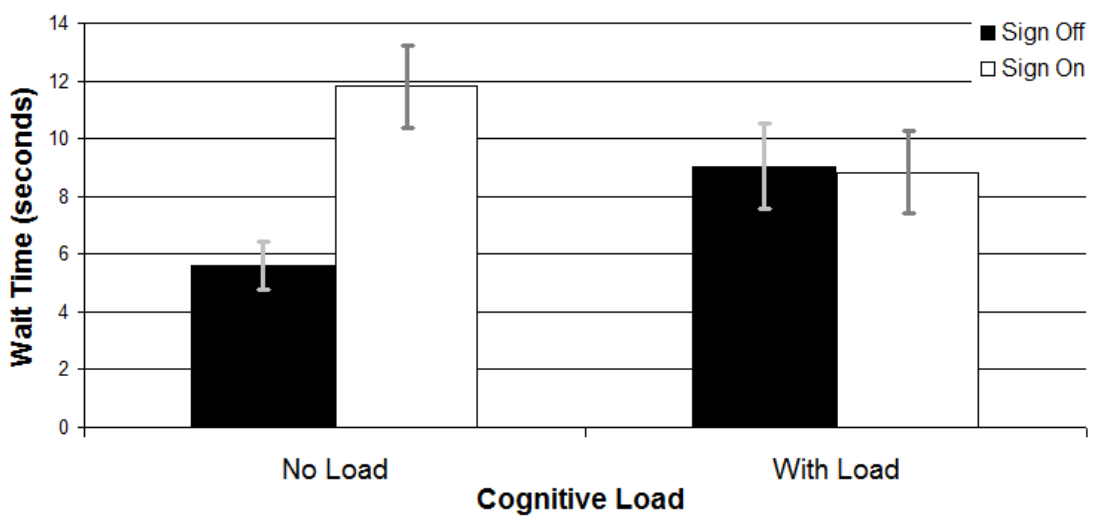

Figure 2. The wait time before crossing the southbound lanes, as a function of Cognitive Load and Sign Presence with standard error bars

Northbound. The analysis of the northbound lanes also revealed a significant main effect of Sign Presence $(\mathrm{F}(1,40)=7.02, \mathrm{p}=.011)$, showing an identical pattern as the wait time for crossing of the southbound lanes (10.8 and 8.6 seconds for Sign On and Sign Off conditions, respectively).

\section{Rejected Gap}

Southbound. The analysis of the rejected gap measure revealed a significant effect of Cognitive Load $(\mathrm{F}(1,44)=4.24, \mathrm{p}=.045)$. Under dual-task, drivers rejected more non-critical gaps (i.e., greater than 6.5 seconds), compared to single-task (.182 and .133 proportion of rejected gaps were non-critical gaps for driving under dual- and single-task conditions, respectively). 
Northbound. The rejected gap analysis when crossing the northbound lanes did not reveal an effect of distraction, however a main effect of Sign Presence was found $(F(1,44)=6.95, \mathrm{p}=$ .012). When the assist system was activated, .14 proportion of all the gaps that participants rejected, were non-critical gaps (i.e., greater than 6.5 seconds), compared to .09 when crossing the intersection without the assist system. As exhibited in a main effect of Age $(F(1,44)=4.89$, $p$ $=.032$ ), older drivers were more likely to reject a non-critical gap when crossing the northbound lanes (.14 proportion of all rejected gaps where non-critical) compared to younger drivers (.08).

\section{Likelihood of Stopping}

Northbound. A significant effect of Cognitive Load $(\mathrm{F}(1,46)=4.51, \mathrm{p}=.039)$, contrary to expectations, showed that drivers completing the dual-task were more likely to make a complete stop at the median (.75 proportion of trials) compared to single-task condition (.69 of trials). This same analysis also revealed a significant main effect of Age $(F(1,46)=7.45, \mathrm{p}=.009)$. Younger drivers were less likely to stop at the median (.63 proportion of trials) than older drivers (.81 of trials). Finally, this analysis exposed a significant interaction between Cognitive Load and Sign Presence factors $(F(1,46)=5.39, p=.025)$. As shown in Figure 3, when completing the singletask, drivers were more likely to stop at the median when the assist system was turned on compared to when it was not activated $(\mathrm{F}(1,46)=7.95, \mathrm{p}=.007 ; \mathrm{M}=.62$ and .74 of trials for Sign off and Sign on conditions, respectively). However, when completing the dual-task, drivers' frequency of stopping at the median was not affected by the state of the in-vehicle CICAS-SSA $(p>.9)$.

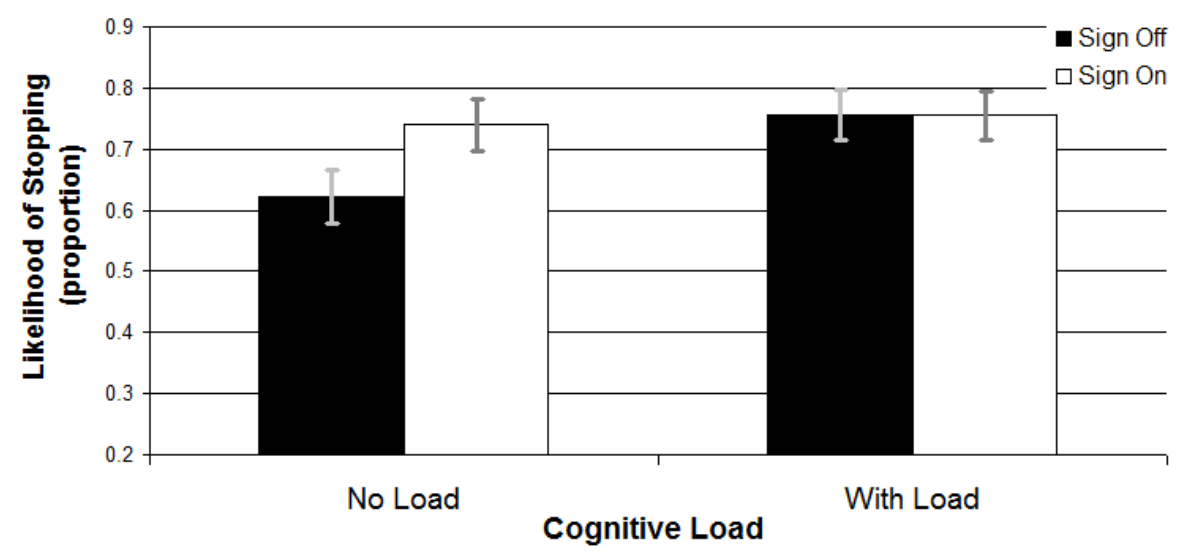

Figure 3. The proportion of intersection crossings in which participants made a complete stop at the median, as a function of Cognitive Load and Sign Presence with standard error bars

\section{Adjusted Time-To-Contact}

Southbound. The analysis of crossing of the southbound lanes revealed a significant main effect of Age $(F(1,46)=4.88, p=.032)$. Younger drivers accepted smaller gaps when crossing the southbound lanes compared to their older counterparts $(\mathrm{M}=5.54$ and 5.79 seconds for younger and older drivers, respectively).

Northbound. The analysis for the northbound lanes also revealed a significant effect of Age $(\mathrm{F}(1,46)=6.11, \mathrm{p}=.017)$, with the same pattern as for the southbound lanes, showing that older driver accepted longer gaps compared to the younger drivers $(M=6.24$ and 5.99 seconds). This 
analysis also exposed a significant 3-way interaction $\mathrm{F}(1,46)=4.72, \mathrm{p}=.035$ ). As illustrated in Figure 4, the Sign Presence and Cognitive Load interaction was significant for older drivers $(\mathrm{F}(1,23)=4.42, \mathrm{p}=.047)$, but not for younger drivers $(\mathrm{p}>.28)$. When performing the dual-task, older drivers accepted longer gaps when the in-vehicle CICAS-SSA was turned on compared to the control, Sign off condition ( $M=6.12$ and 6.36 for Sign off and Sign on conditions, respectively). When completing the single-task, the state of the in-vehicle CICAS-SSA did not impact the gaps older participants accepted.

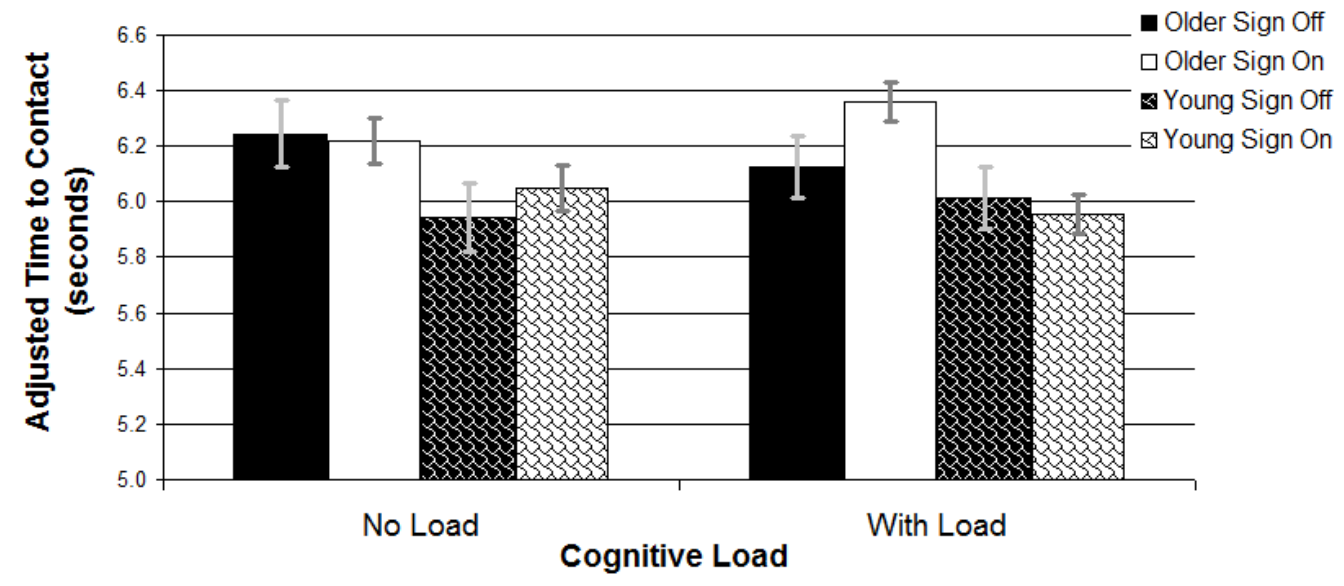

Figure 4. Adjusted time to contact as a function of Cognitive Load, Age and Sign Presence with standard error bars

\section{DISCUSSION}

An important question to ask when incorporating new technology in a vehicle, in addition to its potential benefits, relates to possible discord or even cost when paired with an additional, frequently performed non-driving task (e.g., cell-phone conversation). While the impact of distraction on driving performance during use of collision avoidance systems has been researched (see Kramer et al., 2007), the impact of cognitive distraction on drivers' use of an invehicle information display system (e.g., CICAS-SSA) has not received sufficient attention.

The current results showed that drivers waited longer to cross rural intersections and were more likely to reject a non-critical crossing gap (i.e., greater than 6.5 seconds) when using the invehicle CICAS-SSA. We can interpret these findings as an indication of a more defensive driving behaviour. Interestingly, when completing a concurrent secondary task, drivers were more likely to reject a non-critical gap and make a complete stop at the median compared to distraction-free driving. It is possible that some drivers recognized the inherent risk of engaging in an extraneous task which prompted them to adopt a more conservative driving behaviour.

Of a greater interest to the current research question is the impact of distraction on driver's use of the in-vehicle intersection crossing assist system. Viewed independently, the use of the CICASSSA and driving under an additional cognitive load indicated a stronger emphasis on defensive driving when crossing rural intersections. Viewed in conjunction, does the combination of these two factors have an additive effect, that is, an even greater emphasis on defensive driving? The addition of the secondary task when using the in-vehicle intersection crossing assist system did not change drivers' likelihood of stopping at the median. However, the introduction of the 
secondary task when using the in-vehicle CICAS-SSA, resulted in reduced wait time before crossing the southbound lanes. The wait time under those conditions was still longer compared to the baseline (i.e., without the assist system and distraction-free), suggesting conservative driving, however the additive effect was not present.

Tendency towards defensive driving reveals one aspect of the effect of distraction and in-vehicle CICAS-SSA, but what about the most relevant facet, the ability to select an appropriate gap when crossing the intersection? The in-vehicle CICAS-SSA did not show strong evidence of its effectiveness in choosing appropriate crossing gaps, however the presence of the 3-way interaction showed an interesting pattern. Older drivers, engaged in an additional task were less likely to accept a shorter gap (i.e., shorter ttc when crossing) when the in-vehicle system was activated, compared to system off condition. One possibility is that older drivers more readily recognized the inherent danger of dividing attention between driving and an extraneous task and therefore relied on the in-vehicle CICAS-SSA to cross the intersection when their cognitive resources were strained. However, it is also possible that the older drivers abandoned the secondary task to focus their attention on the primary task of driving (see Kramer et al., 2009).

These results showed that in certain situations drivers engaged in a concurrent non-driving task may exhibit tendency towards conservative driving. One resulting facet of using an in-vehicle intersection crossing assist system includes a greater emphasis on conservative driving. Using an in-vehicle intersection crossing assist system under cognitively demanding conditions did not result in adverse consequences, moreover older drivers appeared to rely more on the in-vehicle assist system when presented with an extraneous additional task.

\section{REFERENCES}

Blaschke, C., Breyer, F., Färber, B., Freyer, J., \& Limbacher, R. (2009). Driver distraction based lane-keeping assistance. Transportation Research Part F: Traffic Psychology and Behaviour, 12, 288-299.

Creaser, J., Manser, M., Rakauskas, M. (2008). CICAS HF3: Sign comprehension, rotation, location, and random gap simulation studies: Final report. St. Paul, MN: Minnesota Department of Transportation.

Kramer, A.F., Cassavaugh, N., Horrey, W., Becic, E. \& Mayhugh, J. (2007). Influence of

Age and proximity warning devices on collision avoidance in simulated driving. Human Factors, 49, 935-949.

Preston, H., Storm, R., Donath, M., \& Shankwitz, C. (2004). Review of Minnesota's rural crash data: Methodology for identifying intersections for intersection decision support (IDS). Minneapolis, MN: Minnesota Department of Transportation.

Rakauskas, M., Creaser, J., Manser, M., Graving, J., \& Donath, M. (2009). CICAS HF4: Validation study: On-road evaluation of the stop sign assist decision support sign. St. Paul, MN: Minnesota Department of Transportation.

Strayer, D.L., Drews, F.A., \& Crouch, D.J. (2006). A comparison of the cell phone driver and drunk driver. Human Factors, 48, 381-391.

Strayer, D.L., Drews, F.A. \& Johnston, W.A. (2003). Cell phone induced failures of visual attention during simulated driving. Journal of Experimental Psychology: Applied, 9, 23-23. 